Théologiques

Théologiques

\title{
Le formel et l'informel : une tension créatrice continuelle
}

\section{Gilbert Renaud}

Volume 3, numéro 1, mars 1995

Symbolique urbaine et foi chrétienne

URI : https://id.erudit.org/iderudit/602419ar

DOI : https://doi.org/10.7202/602419ar

Aller au sommaire du numéro

\section{Éditeur(s)}

Faculté de théologie de l'Université de Montréal

\section{ISSN}

1188-7109 (imprimé)

1492-1413 (numérique)

Découvrir la revue

\section{Citer cet article}

Renaud, G. (1995). Le formel et l'informel : une tension créatrice continuelle. Théologiques, 3(1), 129-152. https://doi.org/10.7202/602419ar

\section{Résumé de l'article}

Posant d'abord le formel et l'informel comme des notions-images qu'il faut penser dans le rapport qui les lie, cet article explore ensuite la forme et l'informe en essayant de montrer leur trajectoire et leur jeu créateur pour enfin s'arrêter sur le mouvement dynamique qui pousse vers la formalisation et suscite l'informel. 
Théologiques 3/1 (1995) 129-152.

\section{Le formel et l'informel : une tension créatrice continuelle}

Gilbert RENAUD École de service social Université de Montréal

\section{RÉSUMÉ}

Posant d'abord le formel et l'informel comme des notions-images qu'il faut penser dans le rapport qui les lie, cet article explore ensuite la forme et l'informe en essayant de montrer leur trajectoire et leur jeu créateur pour enfin s'arrêter sur le mouvement dynamique qui pousse vers la formalisation et suscite l'informel.

\section{Les notions de formel et d'informel}

Formel et informel : deux mots qui ne s'utilisent pas l'un sans l'autre. Deux mots dont il faut, par ailleurs, convenir qu'il est bien difficile de cerner exactement leur définition. Deux mots à la mode - il faut aussi en convenir - utilisés à toutes les sauces sans que personne ne se soucie de les définir de manière rigoureuse pour en faire des instruments théoriques qui permettraient de mieux s'approprier le social auquel ils réfèrent. Deux mots qui peuvent ainsi faire bondir les adeptes de la Science, dès lors qu'ils semblent relever davantage du sens commun et que leur usage - au demeurant d'ailleurs fort courant et répandu dans les conversations des milieux scientifiques - renvoie davantage à la pensée pré-scientifique peu soucieuse de son articulation théorique. Bref, deux mots nébuleux qui échappent - pour l'instant - à la pensée scientifico-positiviste qui se fonde sur la rigueur et la précision du concept servant à l'articulation du cadre théorique. Au contraire, le formel et l'informel appartiennent à ces catégories "molles" et imprécises qui tentent d'approcher le donné avec le souci de le laisser être plutôt que de dicter ce qu'il devrait être. Ainsi, l'utilisation du formel et de l'informel comme catégories d'analyse 
renvoie à une posture épistémologique selon laquelle la connaissance du social - ou, du moins, une certaine connaissance du social - ne peut être complète uniquement dans le cadre d'une pensée qui, à force de se vouloir trop rigoureuse, en vient à réduire le social à ses décrets et à ne plus pouvoir de la sorte rendre compte de ce qui déborde du cadre d'analyse.

Pour être approché, le social requiert une instrumentation diversifiée seule à même de rendre compte de sa complexité et de sa multidimensionnalité. Et l'intérêt suscité par le formel et l'informel traduit bien, à mon sens, le sentiment d'une limite atteinte par une pensée théorique qui s'est vidée de son potentiel de compréhension à la mesure même de son souci de surplomber le social. Le formel et l'informel appartiennent en ce sens à ce nouvel effort de connaissance du social que l'on cherche à approcher d'une manière plus souple pour mieux entendre ce qu'il dit et non ce que dit la théorie. Ainsi posés et utilisés, le formel et l'informel ne constituent aucunement des concepts; ils appartiennent plutôt à l'univers des notions au sens où l'entend Michel Maffesoli. En effet,

sur un objet particulier, le concept est Un, ou tout au moins il s'agence avec d'autres concepts voisins pour former une unité. Il détermine la vérité, ce qui doit être la vérité. Tout ce qui échappe à son emprise est dans l'erreur, et n'a pas droit à l'existence. Voilà schématisée la logique du "devoir-être » qui caractérise l'attitude conceptuelle. (...) C'est pourquoi il vaut mieux opposer à la rigidité du concept la mollesse de la notion. Celle-ci satisfait à notre désir de connaissance tout en relativisant le fantasme du pouvoir qui sommeille dans tout intellectuel. (...) En ce qui concerne la connaissance, l'attitude notionnelle prend acte de l'hétérogénéité, elle donne sur un même objet des éclairages divers, elle indique qu'il est à la fois ceci et cela ${ }^{1}$.

Le formel et l'informel me semblent bien relever de cette "attitude notionnelle ", parce qu'ils participent de ce mouvement de connaissance où s'exprime non pas le souci de déterminer la ou les causes, mais plutôt celui de montrer la tension créatrice de la vie. La vie n'a pas de causes, elle est tout simplement et il convient, par souci intellectuel, d'en rendre compte. C'est bien cette attitude "contemplative", en quelque sorte, que l'on adoptera ici. Le formel et l'informel seront donc utilisés comme

1 M. MAfFESOLI, La connaissance ordinaire. Paris, Librairie des Méridiens, 1985, p. 51. 
catégories notionnelles de pensée qui permettent une large prise de vue sur la vie en général et la vie sociale en particulier.

On comprendra, dès lors, qu'il ne sera donc pas davantage question de tenter de réduire le social à un cadre théorique qui, partant d'une " nouvelle" conceptualisation, enserrerait le donné dans ses décrets. En fait, comme le faisait remarquer Alain Médam dans un numéro de la Reque internationale d'action communautaire consacré aux savoirs en crise, l'heure " n'est plus à la cohérence qui sidère et foudroie la pensée. Elle est à l'étonnement qui torture la pensée et la provoque ${ }^{2}$." L'air du temps semble ainsi appeler un autre parti pris épistémologique qui permet d'échapper «( (à) ce renversement subtil des termes de référence, (où) on en vient ... à cet extrême : ce n'est plus l'objet — dans sa concrétude exubérante - qui détermine et dicte les efforts de la théorie; c'est la théorie, au contraire - dans sa haute sagesse - qui décide de ce qui est digne du titre d'"objet". ${ }^{3}$ " C'est, en quelque sorte, la chance qu'offre le surgissement actuel de cette référence au formel et à l'informel et le chercheur en sciences sociales est, à son propos, invité à adopter une nouvelle attitude qui, plutôt que de chercher encore à saisir et cerner le social, entend maintenant se laisser saisir par celui-ci. La connaissance du social, qui se complaît trop facilement dans le positivisme, n'aperçoit tout simplement plus ce qui déborde de ses cadres si théoriques soient-ils. Ce qui échappe ainsi de plus en plus à la pensée, c'est la vie et comme l'exprimait, encore une fois, Alain Médam: "Ce n'est pas à la vie de conquérir droit de cité dans l'ordre des sciences de la vie; c'est à ces sciences qu'il revient - à ras de sol - de retrouver les émotions de la cité et les mouvements effectifs de la vie ${ }^{4}$."

On le voit bien: le formel et l'informel participent, en fait, d'une posture épistémologique qui s'efforce de renouveler la connaissance du social en se laissant davantage appréhender par celui-ci. Plusieurs seront pourtant tentés de conceptualiser ces notions en espérant - avec les meilleures intentions - en faire des instruments d'analyse et d'action efficaces. Ils les videront ainsi de la richesse et de la fécondité dont elles sont porteuses parce que, tout simplement, elles donnent à penser de multiples manières la vie sociale. C'est, à mon sens, en respectant précisément cette absence de rigueur dans la définition, cette mollesse de précision, qu'on est le mieux à même d'assurer la fécondité d'une image

2 A. MÉDAM, « Des grilles et des vies », RIAC 15/55, (printemps 1986), p. 151.

3 Ibid., p. 152.

4 Ibid., p. 157. 
parlante dont on sent bien qu'elle nous renvoie à quelque chose de fondamental. Mais, du coup, l'on doit renoncer à l'élaboration d'une pensée utilitaire qui permettrait - conformément aux vœux de tous les gestionnaires du social - d'accroître l'emprise que l'on peut avoir sur la vie sociale. Le formel et l'informel nous apprennent, en définitive, que le social - et la vie - échappe continuellement aux impératifs qu'une pensée par trop réductrice voudrait lui imposer. Ils renvoient ainsi au jeu perpétuellement recommencé - et renouvelé - d'une existence jamais achevée et toujours en mouvement. Si le formel et l'informel ont tant de mal à se tailler une place reconnue dans l'univers scientifique, c'est bien parce qu'ils viennent narguer la prétention à l'achèvement et à la maîtrise du social qui a été la principale préoccupation de la pensée moderne. En fait, ils ne servent à rien d'autre qu'au souci et au plaisir intellectuels de se doter de catégories qui permettent de mieux comprendre le donné tout en le laissant être. Ils contribuent de la sorte à bien marquer cette césure dont parle Michel Maffesoli "entre une sociologie positiviste, pour qui chaque chose n'est qu'un symptôme d'autre chose, et une sociologie compréhensive qui décrit le vécu pour ce qu'il est, se contentant de discerner ainsi les visées des différents acteurs ${ }^{5}$. " Le formel et l'informel me semblent bel et bien participer de cette sociologie compréhensive dont la préoccupation est de respecter le vécu en faisant usage de la notion qui " exprime le désir et le souci intellectuel sans pour autant contraindre ou passer à côté, elle ne fait que lier en pointillé ce qui est déjà en morceaux ${ }^{6}$. »

Comme notions donc, le formel et l'informel apportent un éclairage spécifique sur le vécu. Ils constituent, comme je l'ai déjà indiqué, une image parlante (il s'agit là d'un pléonasme, car une image ne vaut-elle pas mille mots?) qui vient associer une chose et son envers. En effet, le formel et l'informel - ou la forme et l'informe auxquels ils se rapportent - sont indissociables (tout comme le sont l'ordre et le désordre, le fonctionnement et le dysfonctionnement); ils constituent un "couple notionnel» où l'un ne se conjugue pas sans l'autre. Dans son introduction à Simmel, Julien Freund signale à cet égard que "l'erreur à ne pas commettre, c'est de concevoir la forme comme un ensemble ou une figure dont tous les éléments seraient consonants. Si la religion ou la politique par exemple sont des formes, elles incluent inévitablement les

M. MAFFESOLI, op. cit., p. 18.

Ibid. , p. 63. 
oppositions et les contestations qui les déchirent ${ }^{7}$." Du formel et de l'informel, nous sommes passés à la forme. Car c'est de forme qu'il s'agit d'abord et avant tout, me semble-t-il. En effet, qu'entendons-nous par formel si ce n'est ce qui participe de la forme? La formalisation, c'est de la forme en travail et le formel, c'est la forme accomplie dans sa rigidité et sa rigueur. Et à chaque forme correspondrait ainsi un informe, à tel point que, selon Alain Médam, "on en finit jamais de l'informe tant il est vrai que les formes en proviennent et s'en nourrissent ${ }^{8}$. "C'est donc dire que le formel et l'informel n'existent que comme rapport qu'il faut penser et voir à l'œuvre de multiples manières.

Et pour en rendre compre le plus adéquatement possible, ne faut-il pas aussi sortir de la langue prescrite par les "manuels de sociologie"? N'est-ce pas "déformer" l'informel - et le formel auquel il se juxtapose - que d'adopter un style par trop conforme aux canons de la scolastique? N'est-ce pas faire injure au donné - pétri d'informel - que de le réduire à une structure formelle de distanciation et d'énonciation? Il semble plutôt que l'heure a également sonné d'une «sociologie qui s'établisse dans un mécanisme constant entre la forme et l'empathie 9 "et qui, par conséquent, ne craint pas de s'adonner aussi à une recherche stylistique trahissant le moins possible la vie dont elle cherche à rendre compte. Il n'y a vraiment rien à faire : dès lors qu'on accepte que le formel et l'informel participent de ces notions-images qui nous aident à penser, plusieurs virages "épistémologiques " s'imposent, à moins que l'on ne décide de se braquer dans la forteresse scientifico-positiviste qui espère - encore - mater le monde et dire - enfin - la vérité de l'informel en "formalisant " un discours à son sujet. Mais alors la notionimage vient rappeler que l'informel ne fonctionne pas par "stratégies", mais par ruses qui jaillissent de tout formel.

\section{La forme technocratique}

Pour suivre le cours du formel et de l'informel et apprécier leur tension créatrice - car c'est bien de cela qu'il s'agit - à sa juste mesure, posons d'abord comme forme le mode de gestion technocratique des sociétés contemporaines. Partons de cette forme massive pour déambuler et montrer le jeu de la forme et de l'informe. Et pour ce faire, procédons

7 J. FREUND, «Introduction », dans G. SIMMEL, Sociologie et épistémologie. Paris, PUF, 1981, p. 39.

8 A. MÉDAM, Le tourment des formes. Montréal, Hurtubise HMH, 1988, p. 218.

9 M. MAFFESOLI, op. cit., p. 30. 
suivant le cheminement proposé par Alain Médam qui, s'insurgeant contre les grilles explicatives, appelait davantage à l'implication comme méthode. "Il s'agit plutôt, écrivait-il, - repartant du milieu du terrain ( comme si l'on avait tout oublié, presque, de ses assurances antérieures) - de théoriser en marchant, en se mouvant, en s'émouvant, à la mesure de ses pas et de ses moyens, au gré des surprises, des désarrois, que le monde réserve à celui qui s'y rend réellement ${ }^{10}$. "Il s'agit, en définitive, d'assumer l'angoisse d'une pensée qui sair que, mises à part ses grilles, elle ne sait pas grand-chose et de se laisser alors pénétrer par une vie toujours énigmatique.

Et que nous amène à penser la "forme " de gestion technocratique des sociétés contemporaines? Elle nous montre que rien n'arrête son cours, qu'une forme peut s'imposer de manière si massive que toute tentative de rectifier son cours semble dérisoire. La forme technocratique semble faire masse au point d'amener toutes les énergies à se conformer à ses règles. Tout se passe, en fait, comme si rien ne pouvait lui échapper; comme si, au détour de chacune de nos actions et de nos volontés de s'en déprendre, elle nous ramenait dans son orbite. La littérature sociologique qui s'intéresse à la domination dans les sociétés modernes l'illustre abondamment. À titre d'exemple, on peut penser aux mouvements sociaux qui semblent porter un renversement ne produisant à terme rien d'autre qu'un perfectionnement de la gestion technocratique ${ }^{11}$. Sans que l'on comprenne comment, la forme moule, pétrit, organise, décrète; bref elle

10 A. MÉDAM, op. cit., p. 153. L'occasion me semble bonne pour préciser ici le lieu à partir duquel sont lancées mes explorations. Professeur à l'École de service social de l'Université de Montréal et m'intéressant à la sociologie du travail social, j'anime également depuis sept ans des groupes de proches de PVVIH (acronyme détestable qui signifie " personne vivant avec le VIH ») dans le cadre d'organismes communautaires de lutte contre le Sida. Depuis un an, je suis président du conseil d'administration du Centre Pierre-Héneault dont la mission est de soutenir et d'aider les proches des personnes séropositives ou sidéennes. C'est à partir de cette «implication " universitaire et communautaite - que je livre donc la présente réflexion.

11 Je pense ici notamment à l'analyse de Robert CASTEL qui a montré mieux que tout autre comment le champ de la santé mentale a été le théâtre de luttes de transformation qui ont conduit au raffinement du mode de gestion technocratique (cf. La gestion des risques, Paris, Minuit, 1981). De même, à propos du mouvement homosexuel, j'ai moi-même soutenu que la lutte pointait - malgré la volonté ou le désir des acteurs - vers une gestion technocratique de l'homosexualité (cf. " Mouvement homosexuel et modernisation technocratique: l'exemple des services sociaux spécialisés pour les homosexuels », RIAC 7/47, (printemps 1982), pp. 136-140. 
"forme et formalise". Elle s'échappe au point d'imposer ses propres finalités souvent à l'encontre même des actions et des volontés. Simmel a bien perçu cette trajectoire de la forme.

Lorsque les relations pratiques, notait-il, et les nécessités poussent les hommes, par la force de leur intelligence, par leur volonté, par l'instinct de transformation et par leurs impulsions sensibles, à élaborer les matériaux de la vie qu'il faut gagner sur le monde, puis à donner des formes déterminées à ces éléments en vue des fins de la vie, et que dans ces formes nous mettons tout cela en ouvre et l'utilisons comme éléments de l'existence - ces forces et ces intérêts se soustraient alors d'une manière singulière à l'utilité au service de l'existence, qui à l'origine les avait fait naître et s'imposer. Des énergies considérables trouvent ainsi leur autonomie en se détachant de l'objet qu'elles avaient formé et ordonné aux fins de l'existence; elles jouent désormais librement pour elles-mêmes et elles créent ou appréhendent une matière qui ne leur servira désormais qu'à leur propre action et réalisation 12 .

Originant de la vie qui l'exige, la forme s'en détacherait donc pour ne plus produire que du formel, c'est-à-dire pour ne plus chercher que son plein accomplissement.

Lancée sur sa trajectoire, la forme ne cherche plus que sa propre réalisation. La forme technocratique ne comprend plus ainsi rien d'autre que sa propre effectuation, rien d'autre que l'accomplissement de ses propres finalités et elle vient dicter ses impératifs dans les moindres recoins de l'existence. Comment, alors, ne pas apercevoir le "triomphe " de la rationalité instrumentale et technique (qui caractérise essentiellement, pourrait-on dire, le contenu de la forme technocratique) que rien ne semble en mesure d'arrêter, qui gagne en extension et en profondeur, comme plusieurs analyses le soulignent? La forme s'est détachée de l'existence. Elle se vautre, en quelque sorte, dans l'autosatisfaction et l'auto-réalisation sans qu'on puisse l'arrêter, même si l'on aperçoit bien qu'elle nous échappe au point de produire une démesure qui nous fascine peut-être. Il semble qu'on ne peut plus lui opposer que notre impuissance à stopper l'accomplissement de ses finalités. La forme technocratique ne cesse ainsi de se "mettre en forme» malgré nos désarrois devant l'irrationnel qu'elle en vient à produire. À cet égard, 
Louis Maheu et Daniel Descent, s'appuyant sur différentes études, ont bien fait ressortir que

toutes tendent à confirmer la rigidité des grands appareils, politiques aussi bien qu'économiques, culturels ou informationnels; toutes dénoncent leur incapacité à limiter leur emprise sur leur environnement externe, leur propension structurelle à manipuler les demandes et les besoins sociaux. Ces grands appareils technocratiques auraient même atteint une forme et un mode de fonctionnement qui les rendrait imperméables à toute velléité d'auto-limitation et d'autorégulation de leurs activités ${ }^{13}$.

En fait, la forme technocratique devient toute-puissante. La rationalité instrumentale se déploie toujours davantage et c'est dans son orbite que le social, par exemple, se conforme de plus en plus à une opération de gestion efficace. Qu'est, en effet, devenue la société, si ce n'est un immense système social qu'il faut rendre le plus opérationnel possible? Michel Freitag avait bien raison de signaler en ce sens que

(le sentiment de l'éclatement du social) coïnciderait plutôt avec l'avènement du social en tant que tel, et la réalisation sans partage de la nature propre, purement "objective" d'abord, et finalement seulement "opérationnelle", de celui-ci. Loin d'éclater, le social manifesterait seulement maintenant, enfin, sa vérité, qui est d'être un pur et simple «système opérationnel " 14 .

Et le système atteint des proportions titanesques. Il s'adjoint toutes les forces, toutes les énergies, tandis qu'il laisse pantois ceux qui voudraient refuser de jouer son jeu. La forme s'étant détachée des fins de l'existence, le social devient gestion et prend figure de planification, de programmation et de prévention, le tout découlant de la mesure de facteurs de risques permettant de cibler des populations en fonction desquelles s'élaborent des techniques d'intervention. Alors que tout le monde sent bien que ce "système " déshumanise, personne ne semble capable de lui échapper. Pire encore, voulant corriger la trajectoire, chacun cherche désespérément à mettre au point le mode d'emploi adéquat. Il faut raffiner la mesure, accumuler davantage de données, mettre au jour de nouveaux facteurs de risque, perfectionner la technique

13 L. MAHEU et D. DESCENT, « Les mouvements sociaux : un terrain mouvant », Nouvelles pratiques sociales, 3/1, (1990), pp. 48-49.

14 M. FreitaG, "Éclatement du "social", ou oubli de la "société"? ", Société 3, (été 1988), p. 10. 
d'intervention, accroître la prévention, bonifier la programmation. C'est ainsi que la forme complète sa formalisation et impose le report constant de l'interrogation sur les finalités d'une organisation de plus en plus formelle. On aurait tort de ne pas suivre Alain Médam lorsqu'il énonce :

en somme et en ce sens, nos formes de vie auraient une vie, seraient en vie. Bien qu'elles soient inertes, les vitalités que nous-mêmes y investissons tandis que nous les habitons feraient qu'elles s'intègrent ces mouvances progressivement ainsi que les contradictions dynamiques dont elles sont porteuses. Bientôt, ces formes nous échapperaient pour partie. Deviendraient, pour une part, autonomes : autoprocessuelles. Se retrouvant précipitées, articulées dans l'univers des formes, comme Simmel l'indique - et bien qu'elles soient nos œuvres - on les verrait imposer leurs exigences vitales, leurs propres tensions dilemmatiques à nos existences. Elles demeureraient des choses mais suffisamment vivantes à leur façon pour que nous ne tenions plus à notre discrétion la vie de ces formes de vie qui, pour leur part, ne nous lâcheraient plus ${ }^{15}$.

Aussi bizarre et impensable que cela puisse paraître, la forme que nous avons créée s'autonomise à un point tel que sa réalisation semble nous transcender.

Comment, en fait, ne pas apercevoir que la forme technocratique impose partout ses finalités? Elle vient agencer et organiser la vie de telle sorte que ses finalités instrumentales s'actualisent toujours davantage. Et c'est ainsi que, par exemple, même l'université - ce lieu pourtant si farouche à l'égard de son autonomie - se transforme de plus en plus en un lieu voué à la réalisation de la forme technocratique. Qui ne voit pas ainsi que les sciences humaines et sociales sont en train d'être aspirées par la forme technocratique? Michel Freitag, dans un article consacré cette fois-ci à la recherche universitaire, montre bien comment cette dernière est de plus en plus vouée à la prise en charge gestionnaire du social. Par le biais de la recherche, l'université se conforme: elle produit de plus en plus de savoirs instrumentaux, techniques et partiels, abandonnant la transmission et le développement d'un savoir synthétique. La recherche est là, pourrait-on dire, ne demandant que sa propre effectuation de mise en forme technocratique de la vie sociale et individuelle. Celle-ci en vient à ne plus consister 
qu'en un immense chantier de recherche et d'intervention, mis au service d'une gigantesque et tentaculaire activité de gestion, avec laquelle la société viendrait à se confondre substantiellement. ... ( La recherche ainsi comprise) devient immédiatement "mode de production" (et non plus de reproduction) de la réalité comprise d'un bout à l'autre comme une artificialité indéfiniment dynamique, qui requiert sa "prise en charge " permanente. Le "réel existentiel " ne disparaît pas pour autant, il se replie sur le domaine subjectif de l'expérience purement privée, intime, mais c'est pour constituer là encore l'objet potentiel de nouvelles recherches (psychologiques, socio-psychologiques, etc. ) conduisant à la constitution de nouvelles modalités de prise en charge professionnelles et organisationnelles, d'un nouveau champ d'intervention, de nouvelles variétés d'experts dans la formation desquels l'université trouvera tout uniment de nouveaux besoins à satisfaire et de nouveaux débouchés à promouvoir. Et ainsi de suite ${ }^{16}$.

Ainsi s'accomplit ce que, pourtant, aucune volonté n'a souhaité ni ne semble vraiment souhaiter actuellement. Aucun esprit n'a voulu que l'université soit ainsi aspirée par des finalités qui lui échappent de plus en plus, et chacun est même prêt à admettre, au contraire, que la pensée pour survivre ne peut s'accommoder des seules contraintes du système de recherche. Il faut bien ainsi admettre que Simmel avait raison de poser que la forme se détache de la vie qui l'a fait naître pour ne plus imposer que ses propres fins.

Tout se passe bien comme si le contrôle de la forme nous échappait de plus en plus, alors même que celle-ci ne cesse de se reproduire. Nous avons créé cette forme, mais nous ne la comprenons plus. C'est elle désormais qui nous tient. Ainsi, toujours à propos de la recherche, Michel Freitag demande

Qui ne voit pas cela physiquement? Les nouvelles technologies de l'information, de la communication, de la cueillette, de la conservation et du traitement des données, sont mille fois plus performantes que les vieilles machines à écrire, les anciens registres, les archaïques classeurs et filières, mais loin de les avoir remplacés, elles les ont multipliés partout, elles ne cessent elles-mêmes de nous envahir dans nos bureaux et laboratoires, et jusque dans nos salons et chambres à

16 M. FREITAG, « La recherche dans l'université et la société : le bateau ne coule pas encore, mais l'eau monte... ", Société 11, (été 1993), pp. 30-31. 
coucher. Partout, elles créent elles-mêmes les besoins qu'il leur faut ensuite satisfaire, partout dans nos universités, dans les centres de recherche et laboratoires, dans les banques, les commerces et les industries, elles se nourrissent des informations qu'elles produisent, elles exigent la production des données qu'elles stockent, elles suscitent l'établissement des réseaux sur lesquels elles multiplient leurs branchements, et cette logique exponentielle, dont l'utilité est prétendument de servir des besoins sociaux, absorbe comme par osmose pour se l'assimiler la moindre molécule de vie sociale encore "libre " à laquelle elle touche ${ }^{17}$.

Comment ne pas voir, en effet, que cette forme nous envahit et qu'elle nous possède, alors que nous croyions la posséder? Comme ne pas voir que cette forme semble exister en dehors de nous tout en nous imposant sa formalisation? Comment ne pas conclure que nous ne comprenons plus ce que nous avons pourtant créé?

Il faut donc bien, encore une fois, concevoir l'inconcevable : la forme s'autonomise des forces qui l'ont fait naître. Dans le cas de la forme technocratique, cela se traduit par ce que plusieurs appellent l'autonomie de la technique, voulant ainsi souligner que la technique répond de plus en plus à ses seules exigences de fonctionnement sans que l'on puisse lui opposer d'autres choix. Aux dires de Guy Ménard et Christian Miquel, la technique se déplierait de plus en plus comme système de toute-puissance mû par

le mythe de l'inéluctabilité de l'essor scientifique et le culte de l'opérativité générale de toutes choses. La technique, devenant système de puissance symbolique auquel tous et chacun participent, s'imposant comme une fin en soi, non soumis à d'autres valeurs, se trouve en quelque sorte justifiée par ce caractère d'inéluctabilité même: technique destin, moderne fatum ${ }^{18}$.

La technique est là, elle fascine et nous amène à ne plus choisir que sa réalisation et son effectuation.

Ibid. , pp. 31-32.

18 G. MENARD et C. MIQUEL, Les ruses de la technique. Le symbolisme des techniques à travers l'histoire, Montréal, Boréal, 1988, p. 336. 


\section{Et l'informe}

Pourtant tout n'est pas si simple, tout n'est pas inéluctablement perdu. Si la forme technocratique s'immisce en tout lieu, il appert également que l'informe la taraude, lui résiste, ruse avec elle. Il convient de se rappeler ici que le formel et l'informel n'existent que l'un par rapport à l'autre; autrement dit, que chaque forme, en même temps qu'elle pourchasse l'informe, suscite le travail de ce dernier. À cet égard, Alain Médam, dans son analyse des formes, note que

voulant nier par la démesure et par l'exaspération de la masse cette présence de l'informe qui les trouble, (les formes) prétendent devenir des mondes par elles-mêmes. Devenir, chacune d'elles, la Forme. Elles ne voient pas que l'informe est indispensable, qu'on ne peut s'en passer et qu'à le réprimer, il se venge; qu'à le reléguer aux pourtours, il n'en occupe pas moins le cœur de la question; qu'à le refouler, il fait problématiquement retour ${ }^{19}$.

Aussi, malgré tous les projets de planification, programmation, prévention, malgré l'ordre parfait auquel elle aspire, la forme percute sur de l'informe qui la fait vivre. En fait, la forme semble tendue vers sa complète réalisation qui signifie, à terme, la mort, tandis que la vie suscite un appel à l'informe qui assure sa permanence. C'est bien, du moins, ce que suggère Michel Maffesoli lorsqu'il signale qu' « en deçà ou au-delà des formes instituées qui toujours existent, il y a une centralité souterraine informelle qui assure la perdurance de la vie en société ${ }^{20}$. "On est bien forcé d'admettre en définitive que la vie semble se lover dans les formes qu'elle suscite tout en les refusant.

Et si, ainsi que je l'ai indiqué, à chaque forme correspond de l'informe, si le formel n'existe qu'en rapport avec de l'informel, il faut à présent nous tourner vers l'informe de la forme technocratique. Or, un des lieux, semble-t-il, où celle-ci s'actualise le plus fortement, c'est l'organisation qui met précisément en œuvre un ensemble de moyens en vue d'atteindre les finalités qui lui sont prescrites. Et l'on sait bien maintenant qu'en vertu de la toute-puissance de la forme technocratique, les finalités avouées risquent d'être détournées - déformées - par les finalités de cette dernière. Ainsi, en va-t-il, par exemple, des services sociaux et de leur organisation. L'on admettra aisément qu'en fait, ce que l'on appelle

A. MÉDAM, op. cit., p. 219.

20 M. MAFFESOLI, Le temps des tribus. Le déclin de l'individualisme dans les sociétés de masse. Paris, Méridiens Klincksieck, 1988, p. 14. 
l'organisation technocratique 21 des services sociaux correspond, en définitive, à une mise en forme d'énergies, une formalisation de celles-ci en vue de concourir aux finalités avouées, qui s'avère, en dernier ressort, contribuer davantage à l'effectuation - de plus en plus stérile par rapport aux finalités avouées - de la toute-puissance technicienne. L'organisation devient folle, pourrait-on dire : devant l'écart grandissant entre les finalités avouées et les résultats obtenus, elle formalise toujours davantage et se comprend de moins en moins. C'est pour cela que l'organisation apparaît comme le lieu par excellence du formel. Et pourtant ... Cette organisation toute formalisante qu'elle soit est traversée par de l'informel. À cet égard, Eugène Enriquez a bien fait remarquer :

Que les organisations n'aient jamais été des lieux uniquement formels, fonctionnels, impersonnels, les théoriciens le savent bien qui ont montré que même dans les bureaucraties les plus rigides existaient des rapports "informels", des regroupements fondés sur les affinités électives, sur la nécessité du travail, sur le contournement des règles, ou sur la défense collective. Toute organisation recèle en son sein des communautés variées, des micro-cultures et se constitue comme un lieu de vie et non comme un simple lieu de travail 22 .

Ainsi faut-il convenir que la vie semble mal s'accommoder des formes qu'elle se donne. L'informe pointe à l'horizon de la forme. Pour le dire comme Alain Médam,

( la forme ) entend localiser un sens - le stabiliser, le particulariser, le parfaire - sans que l'instable, l'insituable, l'impondérable, l'approximatif lui laissent de répit. L'informe la ronge. Cette forme veut conclure et il l'en empêche. Elle veut se clore sur la vérité de sa réponse exclusive tandis qu'il lui montre qu'elle n'a rien d'intangible, celle-ci, qu'elle est toute relative - toute provisoire ${ }^{23}$.

Et d'ores et déjà, malgré la difficulté de la tâche, il faut aussi tenter d'apercevoir cet informe. Où gît-il, demande-t-on? Quel est-il au juste? Peut-il être approché et décrit? Facile, en un sens, de comprendre la forme technocratique : elle est si massive, si incontournable, si tyrannique,

21 Pour une plus grande clarté dans l'exposé, je ne traiterai pas ici des organisations/organismes communautaites dont le rapport à la forme sera analysé plus loin.

22 E. ENRIQUEZ, "Les enjeux éthiques dans les organisations modernes", Sociologie et sociétés XXV/1, (printemps 1990), p. 31.

23

A. MÉDAM, op. cit., p. 220. 
si implacable. L'informe lui oppose son imprécision. Et, a-t-on envie de demander, comment situer l'insituable, comme analyser - et par conséquent, stabiliser par le discours, l'instable, comment apprécier l'impondérable, comment rendre compte de l'approximatif? L'informe ne serait-il pas, en fait, une illusion, un mirage que l'esprit créerait parce qu'il a du mal à accepter les limites que la forme lui impose?

Voilà bien le problème auquel nous sommes confrontés. L'informe échappe vraiment à nos cadres théoriques et à nos discours analytiques. Faut-il admettre alors qu'on ne peut "sociologiser " l'informe, que seule la littérature peut parvenir à l'approcher et à le décrire tant bien que mal? Ce serait alors accepter que la sociologie doit renoncer à parler de la vie, qu'elle n'est qu'une construction intellectuelle qui n'a pas grandchose à voir avec ce dont elle prétend rendre compre, qu'elle ne peut discourir que sur la forme et que, par conséquent, la mort est son véritable fondement. Ainsi, en va-t-il, du moins, de toutes ces analyses qui, en fait, partant d'une intuition juste, s'empressent d'assigner une forme à l'informel qu'elles traquent. Si l'informel s'oppose au formel, s'il en constitue l'envers, si l'informe est ce qui échappe à la forme, alors ne fautil pas renoncer, pour en rendre compte, à le réduire à ces catégories analytiques "formelles" que sont, par exemple, les stratégies et les ressources? Plusieurs, en effet, dans leur désir de lutter contre la forme technocratique - certes de plus en plus aberrante - sont tentés de l'affronter en mettant au jour des stratégies qui conduiraient à la diminution de l'emprise de la forme technocratique sur la vie ${ }^{24}$. Ce faisant, n'entendent-ils pas formaliser l'informel, parce que la stratégie participe forcément de l'instrumentalisation du lien social? C'est bien ce qui permet à la forme technocratique d'ingurgiter, d'englober, de "récupérer " constamment les luttes, ainsi que l'exprime le langage commun. Notre impuissance à stopper le déploiement de la forme technocratique semble tenir précisément à cette obstination d'une confrontation directe et stratégique qui ne peut échapper à la forme, parce qu'elle reprend le contenu et les termes de cette dernière. Rappelonsnous que l'informel ne peut agir qu'en sourdine, qu'il travaille de manière souterraine. Vouloir le ramener à la surface, c'est le trahir. Sa force ne peut être la stratégie, sa puissance est la ruse qui empêche l'enfermement.

\section{4}

Cetre « demande » d'élaboration de stratégies de l'informel ressort souvent chez les étudiants en service social et les travailleurs sociaux que je côtoie. Voyant bien l'informel surgir sur le terrain même de leur travail, ils escomptent, en définitive, une mise en forme rationnelle de celui-ci dans le combat qu'il mène en sourdine et « informellement " pour assurer la perdurance de la vie. 
La forme, pourrait-on dire, s'apparente à la force robuste du chêne, tandis que l'informe appartient à la puissance résistante du roseau dont on sait, depuis La Fontaine, qu'il résiste beaucoup mieux à la violence du vent...

Ainsi en va-t-il également lorsqu'on cherche à situer l'informel comme une "ressource". L'analyse - trop prise par sa grille? - ne voit pas que de concevoir ainsi l'informe, c'est chercher à le formaliser. Une telle conceptualisation participe, en fait, de la forme technocratique qui réduit le social à un système de production mettant en ceuvre un ensemble de ressources-moyens plus ou moins bien organisées ${ }^{25}$. L'informel ne peut qu'échapper à ces catégories et, à vrai dire, seul le fantasme de pouvoir qui sommeille en tout intellectuel peut conduire à une telle perception de l'informel - mais est-ce encore de l'informel? - qui le réduit à l'ordre des ressources, rendant ainsi possible sa mesure - même si celle-ci est qualitative. Difficile d'échapper au paradigme utilitariste qui répond si bien aux finalités de la forme technocratique et qui voudrait bien contribuer à enclore enfin l'informel. Dans la tension incessante qui les lie, le formel ne cherche qu'à mater l'informel qui, en retour, cherche à déstabiliser la forme. C'est pourquoi le langage technocratique propre à la gestion du social n'aperçoit dans l'informel qu'une ressource " complémentaire " qu'il convient de "formaliser et rationaliser " ( un peu d'abord, puis de façon toujours plus massive grâce, notamment, à l'apport - innocent - des sciences sociales qui, se chargeant dans un premier temps d'explorer le terrain procède à une première tentative de formalisation) pour lui faire produire toute sa richesse 26 . Dans l'opération, l'informel disparaît - ou plutôt il ne peut apparaître - , parce qu'il n'appartient pas à l'instrumental : il en est l'envers. Voilà pourquoi l'informel, dès lors qu'on le concevra comme une ressource ou une stratégie, s'échappera du lieu où l'on croit l'avoir assigné à demeure pour apparaître ailleurs.

Des ressources institutionnelles et non-institutionnelles dont on parlait il n'y a pas encore si longtemps, on est passé à une nouvelle formulation qui ne se différencie aucunement de la première, parce qu'en la matière, ce qui prime, c'est la pensée de la " ressource " peu importe qu'on la définisse comme non-institutionnelle ou informelle. Ce faisant toutefois, on a créé l'illusion d'un progrès de la connaissance et d'une meilleure saisie du social. Ainsi fonctionne la pensée qui ne sait plus penser...

26 À cet égard, n'est-il pas significarif de constater l'intérêt conjoint des chercheurs en sciences sociales et des instances étatiques de gestion du social à l'égard de ce qu'ils appeilent les « ressources informelles et communautaires "? Malgré les divergences de leur discours, un même souci les anime : mettre au jour la richesse de toutes les ressources qu'une société peut produire. 
C'est bien à ce point qu'il nous faut le plus oublier nos assurances antérieures. Le risque est grand, en fait, de retomber dans les ornières de la pensée théorico-positiviste qui entend réduire le social à ses grilles en procédant notamment à son instrumentalisation. Le mot est sorti une fois de plus... La forme technocratique se définit par son caractère technique et instrumental. Dès lors, son informe, son envers, surgit de ce qui n'obéit pas à cette instrumentalité. La forme technocratique serait donc rongée par un informe qui échappe à l'instrumental, à la productivité prescrite par la forme. Le rapport tensionnel apparaît dans ce duel entre une forme qui aspire à tout instrumentaliser et un informe qui la mine en introduisant, tel un grain de sable, une force irréductible à l'instrumentalité, une "improductivité » qui vient souligner que la forme n'est pas le tout, une "irrationalité " qui rappelle l'impossible achèvement du social. Si l'on veut donc repérer l'informe dans le brouillard qui l'abrite, il convient de chercher ce qui, au sein de nos sociétés, perdure de résistance à la rationalité instrumentale. À cet égard, Eugène Enriquez nous fournit une première indication lorsqu'il fait ressortir que

le mouvement de la société vers la rationalité intégrale laisse des désirs insatisfaits. ... Au demeurant les hommes résistent à l'instrumentalisation. Ce qui fait de chacun un être humain et social, c'est sa capacité à vivre en état de manque, à formuler de nouveaux désirs, à se laisser aller à son imagination créatrice, qui est à l'origine de toute réflexion et de tout projet, et à nouer avec autrui des relations qu'il devine, avec raison, comme étant essentielles à son intégrité et à sa vie mêmes. ... Il est donc normal qu'une société fondée sur la fonctionnalité et sur la rationalité éveille chez ses membres des désirs de spontanéité, d'actes gratuits, de temps perdu, de passions fortes et de convivialité et qu'une société axée sur la loi du profit et l'élimination des plus faibles fasse ressortir les exigences éthiques 27 .

Il semble bien ainsi que la forme technocratique s'achevant suscite toujours davantage son envers et que cet informe relève, entre autres, de l'irréductible désir qui nous fait être ensemble ${ }^{28}$. L'informe apparaît de

E ENRIQUEZ, op. cit. , p. 28.

28 Le "désir" est bien, en un sens, ce qui échappe à une sociologie qui s'est davantage préoccupée du " besoin ». C'est pourquoi les sociologues bien «formés" éprouvent à son égard un profond malaise devant ce qu'ils considèrent, en fait, comme relevant de l'individualité (et du ressort de la psychanalyse) et non de la socialité. Il 
la sorte chaque fois que, dans la vie sociale, se manifeste du désir, de l'imaginaire, de la gratuité, de l'altérité et que ceux-ci sont agis par une sorte d'impulsion créatrice à être où s'affirme tout simplement une échappée de la forme permettant, par ailleurs, à cette dernière de se nourrir, car, ne l'oublions pas, la forme ne peut vivre sans informe.

Ce à quoi nous renvoie, en définitive, l'informel, c'est à un autre registre de la vie sociale, à ce registre qui n'a pas pris forme technocratique, mais qui la pénètre néanmoins constituant ainsi un rapport tendu. Celui-ci se noue entre deux pôles contradictoires qui travaillent inlassablement l'un contre l'autre et l'un pour l'autre. Tout se passe bien dans la vie sociale moderne comme le suggérait Michel Miranda.

La société moderne, écrivait-il, s'institue en se voulant maîtresse d'elle-même; or, elle se confronte en permanence à une immaîtrisable dynamique sociale. Elle s'affirme comme un espace homogène; alors que le «travail " de l'hétérogène, sous la forme de la différenciation ou du conflit, la taraude sans cesse. Elle se veut totalité adéquate aux individus qui la composent; mais l'intégration sociale, loin de s'effectuer "naturellement», subit certaines formes de résistance. Elle s'imagine transparente, à travers l'écran du politique qu'elle a projeté sur toute forme de sociabilité. Mais dans ses profondeurs et à sa surface même, s'expriment des valeurs qui échappent à sa rationalité, puisqu'elles ne se réfèrent qu'à un ordre symbolique, et ne s'épuisent pas dans une fonctionnalité ou un devoir-être. Dans toute l'épaisseur de la société moderne, brillent des lueurs opaques 29 .

L'informe en ce sens gît dans l'opacité d'un social irréductible à la transparence technocrarique. On l'apercevra effectivement dans cet ordre symbolique qui "dépasse et mine" la raison technique. On le trouvera aussi, semble-t-il, au cœur de ce système technique qui représente pourtant la quintessence de la rationalité instrumentale. En effet,

si la technique froide, écrivent Ménard et Miquel, en arrive à s'imposer, n'est-ce pas en bonne partie dans la rnesure même où, détournée de la rationalité fonctionnelle qui l'a engendrée, elle se laisse traverser d'innombrables lignes de fuite, innerver de courants

serait pourtant grand temps d'élaborer une sociologie fondée sur le désir comme élément incontournable de la vie sociale.

29 M. Miranda, La société incertaine. Pour un imaginaire social moderne. Paris, Librairie des Méridiens, 1986, p. 122. 
chauds qui en font, le plus souvent à l'insu de ses usagers, l'un des nouveaux lieux où se tisse désormais l'être-ensemble contemporain? Tous les micro-rituels évoqués plus haut, quand on y regarde de près, semblent avoir ce rôle de détoumement de la technique de sa fonction purement utilitaire, de rassemblement des individus autour d'une activité commune, d'une passion partagé $\mathrm{e}^{30}$.

Ainsi, en va-t-il, par exemple, lorsque l'intervention professionnelle et technique rompt avec le projet qui l'amène à travailler sur l'autre pour se faire trajet vers l'autre et inaugurer un échange symbolique simplement parce que "le cœur a ses raisons que la raison ignore " et parce que ce visage, sans raison immédiate, m'interpelle ${ }^{31}$. L'informel, c'est cet acte gratuit, ce don que ne peut comprendre la raison utilitaire et qui s'immisce en tout lieu ${ }^{32}$. L'informe, c'est l'arbitraire qui continue de présider à tout lien social mais que la raison technocratique voudrait éliminer parce qu'il contrecarre la pleine réalisation de toute planification et de toute programmation.

L'informel en tant que tel n'existe pas; on s'en souviendra, il n'existe qu'en rapport à une forme. C'est pourquoi l'on peut dire, me semble-t-il, que la communauté - même s'il s'agit là d'une forme - participe de l'informe des sociétés modernes. En effet, la communauté que l'on entendra ici à la manière de Parrick Baudry, c'est-à-dire "comme l'expression de ce qui allie et relie fondamentalement des gens, comme l'expression d'un lien principal (...), et sans quoi il n'y aurait "aucune

G. MÉNARD et C. MiQuel, op. cit., p. 350.

31 David LE BRETON soulignait à cet égard que « le visage de l'autre suscite un sentiment dont il n'est pas toujours aisé de se défaire. Un mouvement de sympathie ou de méfiance, une curiosité, une crainte, parfois un appel, naît de la "première impression", celle dont l'adage dit qu'il est difficile de l'effacer. De tout visage émane une résonance affective, sensible dès le premier regard et qui fournit une sorte d'orientation aux échanges à venir. Cette "impression" est une cristallisation souvent décisive du sentiment que l'autre suscite au vu de son apparence. Elle agit à la manière d'une justification qui se veut raisonnable de l'affectivité éprouvée. Tout contact social est sous l'influence de ce halo de sensibilité qui trouve son ancrage dans le rayonnement du visage. Là se fondent d'emblée les préférences et les exclusives. „ (Des visages. Essai d'anthropologie. Paris, Métailié, 1992, p. 66) La raison professionnelle peut-elle échapper à cet arbitraire?

32 En ce qui concerne le don et sa persistance dans les sociétés contemporaines, cf. Jacques T. GodBout, L'esprit du don. Boréal, Montréal, 1992. 
société"33 ", alimente l'échange symbolique et partant, affirme un ancrage du social dans l'ordre symbolique qui vient pervertir l'instrumentalisation des rapports sociaux. C'est parce qu'il est un des lieux de manifestation de cette communauté que le mouvement communautaire appartient à l'informe. Se fondant largement sur le bénévolat, il est occasion de don, de perte de soi, de création d'échange symbolique, de reconnaissance de l'autre. Et c'est en vertu de ces éléments qui n'appartiennent pas à l'ordre instrumental mais que l'on retrouve dans sa forme que le mouvement communautaire participe de l'informel.

L'informel, c'est donc, pourrait-on dire, une force sociale fluide et imprévisible - une pulsion - qui ruse avec l'ordre instrumental pour briser la monotonie de la forme et qui s'amuse à ébaucher une déviation des finalités auxquelles elle devrait se plier. L'informel de la forme technocratique, c'est donc aussi ce mouvement social - et ces " nouveaux mouvements sociaux» - en tant qu'ils manifestent un bouillonnement de résistance aux impératifs de la gestion technocratique et ne cessent de rappeler l'existence de la personne dont l'identité est irréductible à celle prescrite par les appareils en vue de leur seul fonctionnement efficace. Le mouvement social relève de l'informel en autant et seulement parce qu'il est cristallisation et manifestation du sujet qui refuse son objectivation. Non pas donc d'abord parce qu'il est lutte ouverte et encore moins parce qu'il est organisation de résistance, mais parce que s'y affirme la quête d'un rassemblement où prime l'être plus que le faire.

À trop me suivre, on croirait presqu'avoir - enfin - cerné l'informe. À trop vouloir le visualiser, on risque ainsi d'acquérir l'assurance de celui qui pense avoir réussi à dompter l'informel. Pourtant, celui-ci n'est d'aucun lieu, d'aucun espace, il est plutôt ce chaos fondateur qui surgit en tout espace et tout lieu. Toutes ces tentatives de décrire l'informel ne sont-elles pas déjà trop précises en sorte qu'il leur échappe? Ne suis-je pas tombé dans ce que, moi-même, je dénonce? Ne suis-je pas en train de donner forme à ce qui, de toutes les manières, échappe à la forme? Il convient donc de se remémorer que l'informe est souterrain, qu'il est mystérieux, qu'il agit en sourdine, qu'il ruse, qu'il appartient au règne de l'opaque, qu'il taraude plus qu'il ne résiste, bref qu'il ne se laisse pas saisir et qu'il faut trouver le moyen, malgré tout, d'en rendre compte et ce, en le trahissant le moins possible. Il faut donc garder en tête que toutes mes indications ne sont qu'approximations de l'informe et comprendre que 
l'informel, c'est ce qui perdure de la genèse, de l'impulsion première et bouillonnante qui a donné naissance à la forme, du tumulte des premières heures qui resurgit de manière larvée ou violente lorsqu'à force d'avoir été trop réprimé, il ne trouve plus que cette voie d'expression. On n'oubliera donc pas qu'en matière d'informe, on ne peut qu'indiquer ce vers quoi il faut tourner le regard pour percevoir sa rumeur bouillonnante. Et s'il est des analyses aidantes en ce qu'elles permettent d'établir une analogie entre des phénomènes différents, c'est peut-être vers celles du sacré qu'il faut s'orienter. En effet, s'inspirant de Bataille, Guy Ménard notait que

le sacré, pour ( celui-là ), c'est essentiellement - et, en un sens, très simplement - le bouillonnement prodigieux, le déchaînement exubérant aveugle et violent de la vie sous toutes ses formes, déchaînement que l'ordre des choses, pour durer, doit de quelque manière enchaîner, endiguer. Se trouve par là définie, en contrepartie, la sphère du profane: le profane, dans cette perspective, ce sont justement ces "digues" qui viennent enchaîner, harnacher le prodigieux déchaînement de la vie, et sans lesquels la vie humaine (en tant qu'humaine) serait impossible ${ }^{34}$.

Il en va de même pour la forme et l'informe et comme la religion, pour Ménard, est gestion des rapports entre les deux sphères, on pourrait penser que les organisations constitueraient le lieu de gestion des rapports entre le formel et l'informel.

\section{Le mouvement dynamique}

On s'en est probablement aperçu : en tentant d'approcher l'informe, notre réflexion est allée vers le rapport qui l'unit à la forme. Toujours revient, en fait, ce rapport qui est mouvement dynamique de création et c'est ce dynamisme qu'il importe à présent de mieux comprendre. Et, puisqu'en cours de route, nous avons cheminé vers le mouvement social et communautaire tout en sentant bien que le terrain y devenait glissant, parce qu'à n'en pas douter, il n'est pas à l'abri du formel, c'est néanmoins à partir de lui que nous tenterons de mieux percevoir ce mouvement de tension dynamique.

Il faut commencer par se défaire d'une analyse trop répandue qui, dans le cas des services sociaux par exemple, associe l'informel au secteur communautaire et le formel au secteur public. Découpage simpliste qui ne culture au Québec. Figures contemporaines du sacté. Montréal, Fides, 1986, pp. 61-62. 
résiste pas à l'analyse. D'abord parce que, ainsi que je l'ai déjà dit, toute organisation quelle qu'elle soit ne peut vivre sans que s'y maintienne de l'informel. On le sent bien: une organisation trop formelle devient vite étouffante et ne peut plus parvenir à susciter les énergies qui assurent son maintien. Quel travailleur social ne sait pas, en outre, que pour s'établir, la relation professionnelle nécessite un minimum d'informel? À cela s'ajoute surtout le fait que le secteur communautaire est loin d'échapper à la formalisation. Dans leur présentation du numéro de Sociologie et sociétés consacré à la gestion du social, Louis Maheu et Jean-Marie Toulouse font bien ressortir que "le social qui se construit n'est pas à l'abri non plus de nettes tendances à l'institutionnalisation et à la professionnalisation de l'action collective et des mouvements sociaux ${ }^{35}$." Dès lors qu'il se construit, le social ne peut que prendre forme et le mouvement communautaire, certes loin d'être homogène dans ses organisations, n'échappe pas à sa technocratisation, c'est-à-dire à sa formalisation technocratique. Il faut être vraiment extérieur aux organismes communautaires pour ne pas voir à quel point ils viennent précisément formaliser l'énergie informelle qui a pu leur donner naissance.

À cet égard, on n'a qu'à suivre le bénévolat souvent présenté comme la "forme » par excellence des ressources informelles que l'on retrouve en si grand nombre dans le secteur communautaire. On pourra facilement constater que le bénévolat n'a rien d'informel et que, pour lui permettre d'atteindre ses finalités, il doit être organisé. D'ailleurs, ne s'institue pas bénévole qui veut : les bénévoles sont maintenant de plus en plus formés par les organisations qui les utilisent. Belhassen Redjeb a su bien montrer comment le bénévolat s'est modernisé contribuant ainsi de plus en plus à la gestion technocratique du social ${ }^{36}$. Le bénévolat n'a rien d'informel et l'on a donc bien raison d'en faire une ressource. L'informel peut certes advenir dans le bénévolat, tout comme il peut surgir aussi dans la relation professionnelle. Il est ce souffle chaud, cette "âme" ( qu'on me pardonne, je n'ai pas d'autre mot), cette gratuité généreuse, cette pulsion vers l'autre, cette passion du lien social qui émerge sans raison dans l'arbitraire de la rencontre. Il introduit une autre dimension dans la vie sociale en y mettant - de manière larvée ou violente - une sorte de butée qui énonce

L. MAHeU et J.-M. Toulouse, «Présentation. Gestion du social et gestation du social », Sociologie et sociétés, XXV/1, (printemps 1993), p. 21.

36 B. REDJEB, «Du bénévolat au néo-bénévolat », Nouvelles pratiques sociales, $4 / 2$, (automne 1991), pp. 59-79. 
l'impossible achèvement souhaité par la forme. Au bout du compte, le bénévolat apparaît comme la forme organisée de la pulsion au don qui est au fondement du lien social. Il semble, en quelque sorte, originer d'un informel bouillonnant qui cherche le chemin de l'autre dans sa souffrance, mais qui, pour être, doit emprunter le chemin de sa formalisation.

Mais alors, demandera-t-on, ne faut-il pas aussi être aveugle pour ne pas voir la distance qui sépare le bénévolat de l'action professionnelle et celle qui existe entre un groupe communautaire et une organisation publique de services sociaux? Certes, conviendra-t-on, l'organisme communautaire met en ouvre une certaine formalisation du social, mais de là à tout ramener sur le même plan, il y a un pas qu'on ne saurait franchir. Et l'on aura raison. Par rapport à la relation professionnelle, le bénévolat présente une plus grande souplesse, une générosité supérieure, une disponibilité plus large, une ouverture à l'autre plus profonde, bref, il se rapproche beaucoup plus de la relation sociale primaire et se vit davantage comme trajet que comme projet, ce qui amène précisément plusieurs à la situer dans l'informel. Et l'organisme communautaire qui lui permet de se manifester présente par rapport au réseau public les mêmes caractéristiques. Qu'est-ce à dire au plan de la forme? Freund fournit une première indication lorsque, commentant toujours Simmel, il signale qu' " il y a des formes massives et des formes presque indistinctes, il y a des configurations solidement constituées et d'autres qui sont en perpétuel devenir et qui sont la trame de la vie quotidienne des êtres ${ }^{37}$." Tout semble bien relever de la masse. Les formes nous pétrissent, mais elles ne présentent pas toutes la même rigueur, la même masse, le même pouvoir d'attraction et de conformation des énergies qui s'y déploient. Entre le bénévolat et la relation professionnelle, entre le secteur communautaire et le réseau public, existe une différence de masse et en s'inspirant des lois de la gravité, on peut comprendre que la masse variant, l'attraction - la conformation - se modifie de l'un à l'autre. C'est donc la plus grande légèreté de la forme qui donne à l'un sa plus grande perméabilité au surgissement de l'informel, tandis que la masse de la forme technocratique rend plus difficile de s'échapper de son orbite et que cette forme requiert pour sa transformation une somme considérable d'énergies. Tout semble, en fait, se passer comme si existait une masse critique à partir de laquelle la forme s'autonomiserait des énergies qui lui ont donné naissance. 
Dès lors, il est possible de penser que le mal-nommé secteur « informel » constitue une forme encore proche du tumulte bouillonnant qui lui a donné naissance, une forme en cours de réalisation, moins achevée et tourbillonnant encore autour de son impulsion créatrice. Une forme qui n'a pas encore basculé dans sa complète autonomisation et qui, par conséquent, ne cherche pas encore que sa propre effectuation et ne dicte pas que ses propres fins. Ainsi se comprendrait la différence entre les secteur public "formel " et le secteur communautaire "informel ", de même que la différence se mesurant dans le temps entre les débuts de l'organisation et son développement. Si l'on songe, par exemple, au secteur communautaire, cette transformation est notamment visible dans les réaménagements de l'espace, dans l'acquisition des moyens techniques qui propulsent l'organisation dans un autre stade de développement, dans l'organisation et la division du travail, etc. Le chaos originel prend toujours davantage forme, il disparaît de plus en plus étant aspiré par la masse technocratique. Alain Médam a donc bien raison: "L'informe, c'est aussi la genèse : le lieu des recommencements agités, des reformulations opiniâtres, des re-formations ${ }^{38}$."

Ce que l'on aperçoit, en fait, dans tout cela, c'est d'abord le rapport dynamique constant qui s'établit comme trajet entre deux pôles contradictoires se contaminant l'un l'autre. L'informe prend forme qui s'en nourrit. Et à ceux qui jubilent presque de découvrir les "vertus " de cet informel qu'ils nomment mal et qu'ils espèrent régenter pour le faire régner, il convient de rappeler, à la suite de Médam, que

la vie fait un choix. Certes, elle endure tout le tragique de cette tension qui l'écartèle entre forme et informe - entre volonté de se définir et désir de se laisser prendre par le chaos fascinant - mais néanmoins opte pour les formes, renonçant pour cela à toute une part de ce qu'elle est. Elle se rationalise. Elle se réfrène. Bien qu'elle se révolte périodiquement contre les contraintes qu'elle s'impose, bien qu'elle revienne alors à l'impulsion première, primordiale, parce que ses raisons hautement affirmées ne lui semblent pourtant pas lui suffire, elle n'en finit pas de se résoudre chaque fois à l'empire des limitations constructives mais sévères qu'elle se donne ${ }^{39}$.

Ce qu'il faut avant tout comprendre - et accepter -, c'est que la vie sociale - excède toute tentative de rationalisation, qu'elle cherche à y 
échapper tout en devant s'y lover pour être. Comprendre que ce qui apparaît, ce n'est pas tant d'abord la forme et encore moins l'informe, mais le mouvement perpétuel qui va de l'un à l'autre. S'évanouissent alors nos fantasmes de structures, de causes et de déterminations qui font place à cette image - qu'utilisent les physiciens pour rendre compte de l'organisation vivante - du " tourbillon de liquide, qui se fait et se défait, dont la forme reste à peu près stable, à la fois contre et grâce à des perturbations aléatoires, imprévisibles, qui maintiennent ce tourbillon tout en le détruisant, et le détruisent tout en le maintenant ${ }^{40}$."

En matière de formel et d'informel, de forme et d'informe, il faut accepter d'être bousculé, désarçonné; il faut penser l'impensable, concevoir l'inconcevable. Il faut accepter de se laisser dépasser et pour peu qu'on les prenne au sérieux, d'abandonner les prêts-à-penser qui sont le royaume de la déformation du social. Il faut se laisser aller à ces "notions-images" si peu prisées par la communauté scientifique mais qui, étant si parlantes, permettent de mieux comprendre la vie sociale parce qu'elles appartiennent à ce savoir "instinctif et expérienciel " qui nous est donné et à partir duquel on sent que la réalité dépasse la fiction... Mais que faire de tout cela, demandera inévitablement celui qui se préoccupe de l'efficacité d'une pensée et qui, fort de ses grilles, entend les " appliquer» sur le social? S'émerveiller tout simplement de ce que, malgré le désenchantement du monde, l'enchantement perdure et se réjouir du fait que l'on puisse y contribuer un tant soit peu.

40 H. A TLAN, Entre le cristal et la fumée. Essai sur l'organisation du vivant. Paris, Seuil, 1979, p. 281, dans M. MIRANDA, op. cit. , p. 205. 